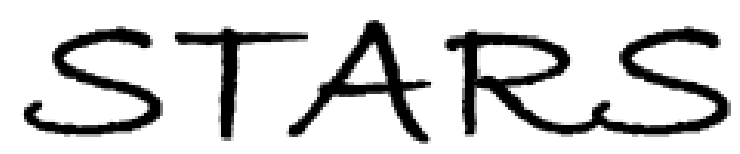

University of Central Florida

STARS

$1-1-2009$

\title{
Diffusion mediated photoconduction in multiwalled carbon nanotube films
}

\author{
Biddut K. Sarker \\ University of Central Florida
}

M. Arif

University of Central Florida

Paul Stokes

University of Central Florida

Saiful I. Khondaker

University of Central Florida

Find similar works at: https://stars.library.ucf.edu/facultybib2000 University of Central Florida Libraries http://library.ucf.edu

This Article is brought to you for free and open access by the Faculty Bibliography at STARS. It has been accepted for inclusion in Faculty Bibliography 2000 s by an authorized administrator of STARS. For more information, please contact STARS@ucf.edu.

\section{Recommended Citation}

Sarker, Biddut K.; Arif, M.; Stokes, Paul; and Khondaker, Saiful I., "Diffusion mediated photoconduction in multiwalled carbon nanotube films" (2009). Faculty Bibliography 2000s. 2092.

https://stars.library.ucf.edu/facultybib2000/2092

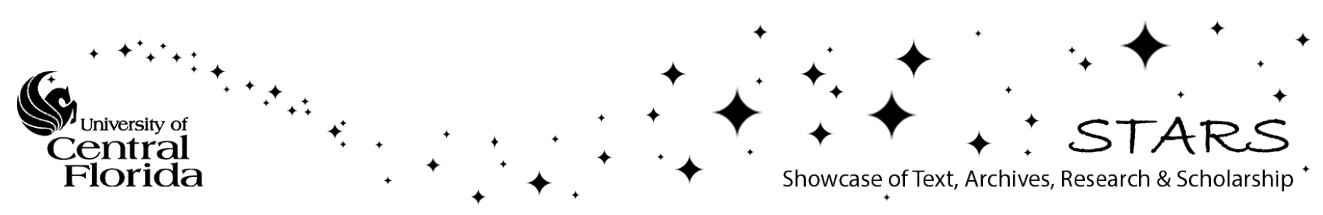




\section{Diffusion mediated photoconduction in multiwalled carbon nanotube films}

Cite as: J. Appl. Phys. 106, 074307 (2009); https://doi.org/10.1063/1.3243335

Submitted: 17 July 2009. Accepted: 09 September 2009. Published Online: 13 October 2009

Biddut K. Sarker, M. Arif, Paul Stokes, and Saiful I. Khondaker
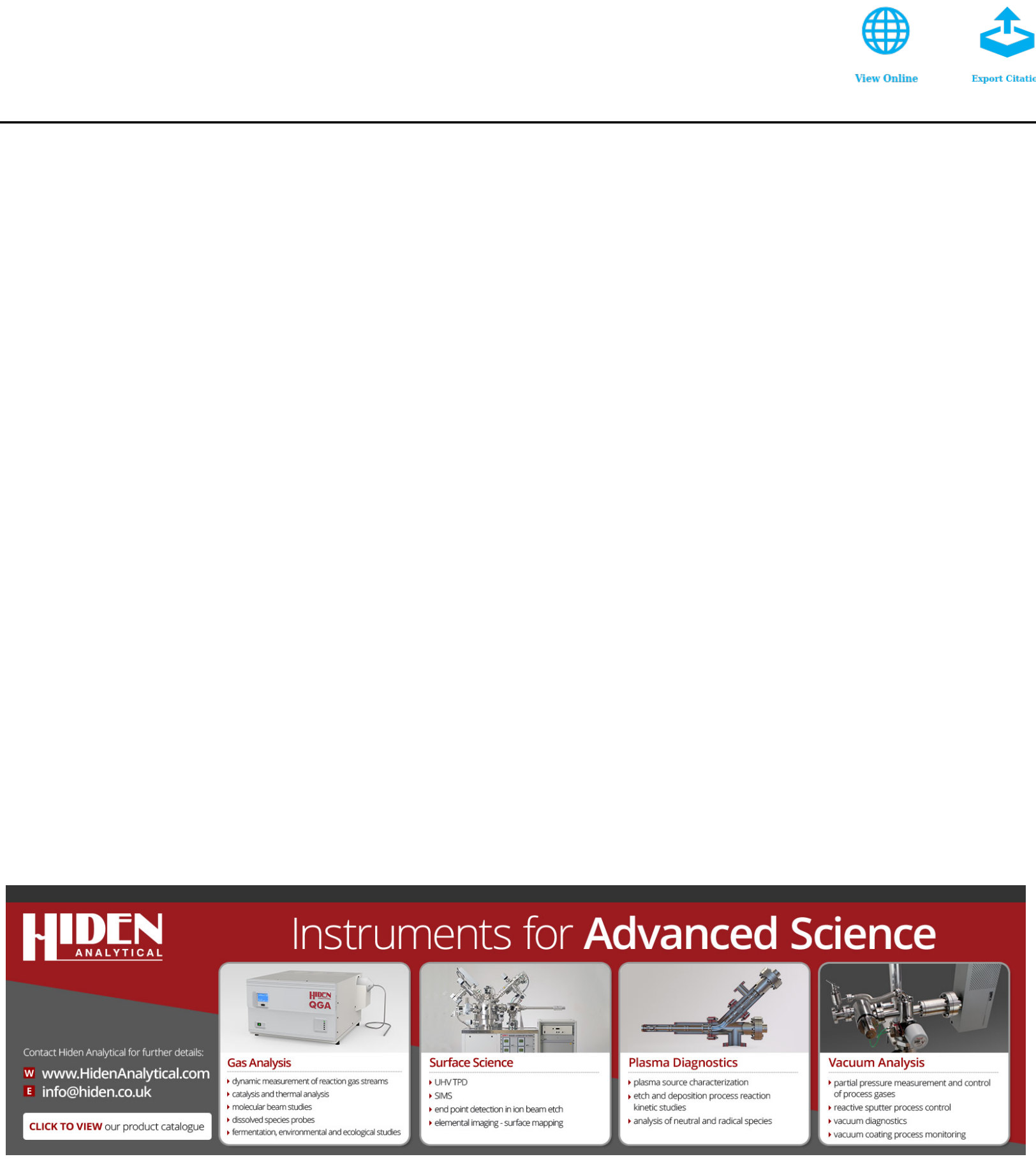


\title{
Diffusion mediated photoconduction in multiwalled carbon nanotube films
}

\author{
Biddut K. Sarker, M. Arif, Paul Stokes, and Saiful I. Khondaker ${ }^{\mathrm{a})}$ \\ Nanoscience Technology Center and Department of Physics, University of Central Florida, Orlando, \\ Florida 32826, USA
}

(Received 17 July 2009; accepted 9 September 2009; published online 13 October 2009)

\begin{abstract}
We present a near infrared photoresponse study of large area multiwalled carbon nanotube (MWNT) films with different electrode separations. We show that the photocurrent strongly depends on the position of the laser spot with maximum response occurring at the metal-film interface. The time constant of dynamic photoresponse is slow and increases with increasing electrode separations. The photoconduction mechanism can be explained by the Schottky barrier modulation at the metal-nanotube film interface and charge carrier diffusion through percolating MWNT networks.

(C) 2009 American Institute of Physics. [doi:10.1063/1.3243335]
\end{abstract}

\section{INTRODUCTION}

Carbon nanotubes (CNTs) are considered to be promising building blocks for nanoelectronic and optical devices due to their special geometry, high electrical conductivity, and exceptional mechanical and optical properties. ${ }^{1,2}$ In particular, photoresponse studies of pure CNT films and CNT/ polymer composites have attracted tremendous attention because of their easy processability at macroscopic dimensions and promising applications in optoelectronic devices. ${ }^{3-15}$ However, these studies also generated considerable debate about the origin of photoconductions in CNT films.

Itkis et ll $^{3}$ found that in single-walled CNT (SWNT) film, the photoresponse was due to a bolometric effect, a change in conductivity due to heating of the SWNT network, while Levitsky and Euler ${ }^{7}$ showed that in SWNT film, molecular photodesorption is responsible for the change in conductivity upon near infrared (NIR) source illumination. Pradhan et al., ${ }^{8}$ in SWNT/polymer composite, also found that the photoresponse is bolometric. However, in these measurements, the size of the electrodes was either smaller than the laser spot size or the laser was positioned in the middle and authors did not check the effect of contacts. Further investigations of photoresponse in macroscopic SWNT films ${ }^{9-14}$ with large electrode separation have shown that the photocurrent generation depends on the position of the laser spot and maximum photoresponse occurs at the metalSWNT film interface, which can be explained by the Schottky barrier modulation model. ${ }^{9}$

Although there are several studies of photoresponse in SWNT films, there are only a few experimental reports on the photoresponse of multiwalled CNT (MWNT) films. ${ }^{4,16}$ It should be noted that unlike SWNT, the band structure of MWNT is very complex and difficult to model and the transport properties of MWNTs are usually diffusive. ${ }^{17}$ The mechanism for photoresponse in MWNT is not well understood and calls for further experimental and theoretical studies. In particular, experiments of MWNT films with different electrodes separation can be useful in understanding the pho-

\footnotetext{
a) Author to whom correspondence should be addressed. Electronic mail: saiful@mail.ucf.edu.
}

toconduction mechanisms in these films. Moreover, MWNT films can be advantageous for practical applications over SWNT films as they can be mass produced cheaper than SWNT $\left(\sim \$ 500 / \mathrm{g}\right.$ of SWNT versus $<\$ 10 / \mathrm{g}$ of MWNT). ${ }^{18}$

In this paper, we present a NIR photoresponse study of MWNT films with different electrodes separation to investigate the photoconduction mechanism in MWNT film. We observed strong dependence of the photocurrent on the position of laser spot with maximum photoresponse occurring at the metal-MWNT interface. We also show that the time constant of dynamic photoresponse at the metal-film interface depends on the electrodes separation and that the time constant increases from 0.35 to $5.3 \mathrm{~s}$ as the electrodes separation increases from 2 to $50 \mathrm{~mm}$. While the photocurrent generation can be explained by a Schottky-type barrier modulation at the metal-MWNT film interface, the slow time response can be described by a model of the diffusion mediated conduction of charge carriers through many interconnected MWNTs.

\section{EXPERIMENTAL DETAILS}

MWNT films were prepared using a drop cast technique. The MWNTs with a purity of $>95 \%$ were purchased from Nanolab. The diameter and length of the as-purchased MWNTs are 10-20 $\mathrm{nm}$ and 5-20 $\mu \mathrm{m}$, respectively. MWNTs were dispersed into 1,2 dichloroethane and sonicated for 3-4 h in water bath kept at constant temperature of $10-15{ }^{\circ} \mathrm{C}$. The concentration of the solution was $1 \mathrm{mg} / \mathrm{ml}$. After dispersion, an appropriate amount of solution was drop cast onto a glass slide to make a thin layer of film. The slide was kept on a hot plate at around $40-50{ }^{\circ} \mathrm{C}$ for $10-15 \mathrm{~min}$ to evaporate the solvent, after which another layer of thin film was deposited. The resulting film had a thickness of $\sim 40 \mu \mathrm{m}$. Finally, conducting silver paste was used to make pairs of electrodes of various separations $d=2,3,5,10,20$, $25,40,50 \mathrm{~mm}$ with a fixed channel width of $25 \mathrm{~mm}$. Figure 1(a) shows a field emission scanning electron microscope image of one of our films and Fig. 1(b) shows an optical micrograph of one of the samples with 10 and $50 \mathrm{~mm}$ electrode separations. 

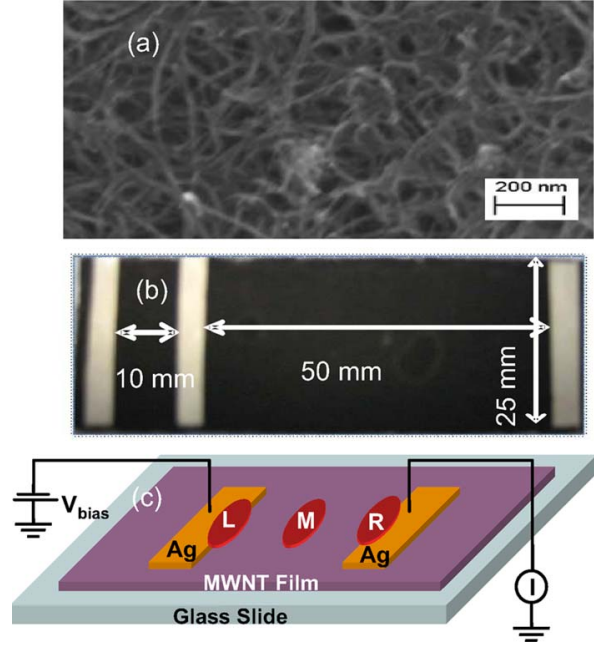

FIG. 1. (Color online) (a) Scanning electron micrograph of a MWNTs film. (b) An optical micrograph of one of the samples showing two pairs of electrodes. (c) Schematic diagram of the device and electric transport measurement setup. The spacing between the electrode varied from $2-50 \mathrm{~mm}$, and the wavelength of NIR source is $808 \mathrm{~nm}$. L, M, and R mark the position of the laser.

Figure 1(c) shows a schematic diagram of a final device and the electrical transport measurement setup. The room temperature dc charge transport measurements of the MWNT films was carried out in a probe station using a standard two-probe technique both in the dark and under illumination by a NIR laser spot positioned at three different locations: L corresponds to illumination on the left electrode/film interface, $\mathrm{M}$ is between the electrodes in the middle of the sample, and R is the right electrode/film interface. The NIR source consists of a semiconductor laser diode with a peak wavelength of $808 \mathrm{~nm}(1.54 \mathrm{eV})$ driven by a Keithley 2400 . The spot size of the laser was approximately 10 -mm-long and 1-mm-wide. The photointensity was monitored with a calibrated silicon photodiode (Thorlabs S121 B). Unless mentioned otherwise, the power intensity of the laser was $\sim 4 \mathrm{~mW} / \mathrm{mm}^{2}$ at the distance it was placed from the sample $(\sim 20 \mathrm{~mm})$. The photocurrent was calculated by subtracting the dark current from the current under NIR source illumination. The bias voltage was fixed at $1 \mathrm{mV}$. Data were collected by means of LABVIEW program interfaced with the data acquisition card and current preamplifier (DL instruments, Model 1211) capable of measuring subpicoampere signal.

\section{RESULTS AND DISCUSSIONS}

Figure 2 shows a typical photoresponse curve for one of our MWNT films with electrodes separation $d=10 \mathrm{~mm}$, where we plot photocurrent as a function of time $(t)$ when the laser spot was positioned at $\mathrm{L}, \mathrm{M}$, and $\mathrm{R}$, and was turned on and off every $100 \mathrm{~s}$ interval. It can be seen that the photocurrent strongly depends on the position of the laser spot. When illuminated at position L, there is an increase in photocurrent. When shined at position $\mathrm{M}$, there was a much smaller photocurrent generation, whereas position $\mathrm{R}$ shows a decrease in photocurrent when illuminated by the NIR source. For this sample, the photocurrent at position $M$ is

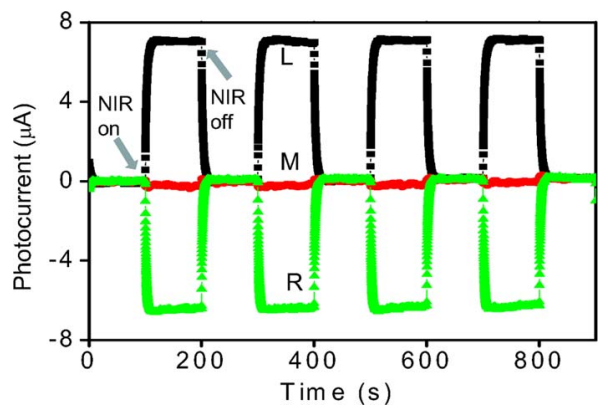

FIG. 2. (Color online) Representative photocurrent as a function of time for a film with $10 \mathrm{~mm}$ electrode separation under NIR source illumination at positions $\mathrm{L}, \mathrm{M}$, and $\mathrm{R}\left(V_{\text {bias }}=1 \mathrm{mV}\right)$. The IR laser is turned on and off at every $100 \mathrm{~s}$ interval.

$-0.19 \mu \mathrm{A}$, while it is 7.08 and $-6.45 \mu \mathrm{A}$ for position $\mathrm{L}$ and $\mathrm{R}$, respectively. Compared to the middle position, the magnitude of the maximum photocurrent enhancement at the metal-nanotube interface is about 36 times. It can be seen that the on and off current is completely reproducible over several cycles. Similar position-dependent behavior of the photocurrent has been observed in all our samples with electrode separations ranging from 2 to $50 \mathrm{~mm}$. The large enhancement in photocurrent at the metal-CNT interface can be described by Schottky barrier model. ${ }^{9}$ When the laser is shined at the left metal-nanotube interface, photons are absorbed by CNTs, which in turn create excitons (bound electron-hole pair). Some of these electrons have enough energy to overcome the barrier potential by tunneling or thermal emission and fall into metal electrode leaving holes in the nanotube film. This induces a separation of electrons and holes at the interfaces and creates a local electric field. Therefore, a positive photocurrent generates at this interface. On the other hand, when the laser shines at right interface, the separation of electrons and holes also generates a local electric field, but in the opposite direction than that of left interface. Therefore the photocurrent is negative with almost the same magnitude as of the left electrode. A very small negative photocurrent is seen at position $\mathrm{M}$ because of the finite width (10-mm-long and 1-mm-wide) of the NIR source and a small positioning error as it was positioned manually, for which the position M may be little closer to R. Therefore some net energetic excitons could travel to the right electrode, generating a separation of a few electrons and holes and creating a very small negative photocurrent.

Figure 3(a) shows a representative plot of photocurrent versus time when illuminated at position $\mathrm{L}$ for another sample with electrode separation of $25 \mathrm{~mm}$ for a few laser intensities $\left(2.37,3.33,4.01\right.$, and $5.68 \mathrm{~mW} / \mathrm{mm}^{2}$ from bottom to top). The intensity of laser light was changed by changing the height between the sample and laser source. The plot is shown for two cycles of the laser being turned on and off at every $100 \mathrm{~s}$ interval. In Fig. 3(b), we plot the photocurrent versus laser power intensity for the same sample shown in Fig. 3(a) for all the laser intensities. The solid line is a linear fit of the data, which shows that the photocurrent increases linearly with intensity. A similar observation was reported for SWNT films. ${ }^{7}$ When the intensity of the laser light is higher, more photons are absorbed by the 

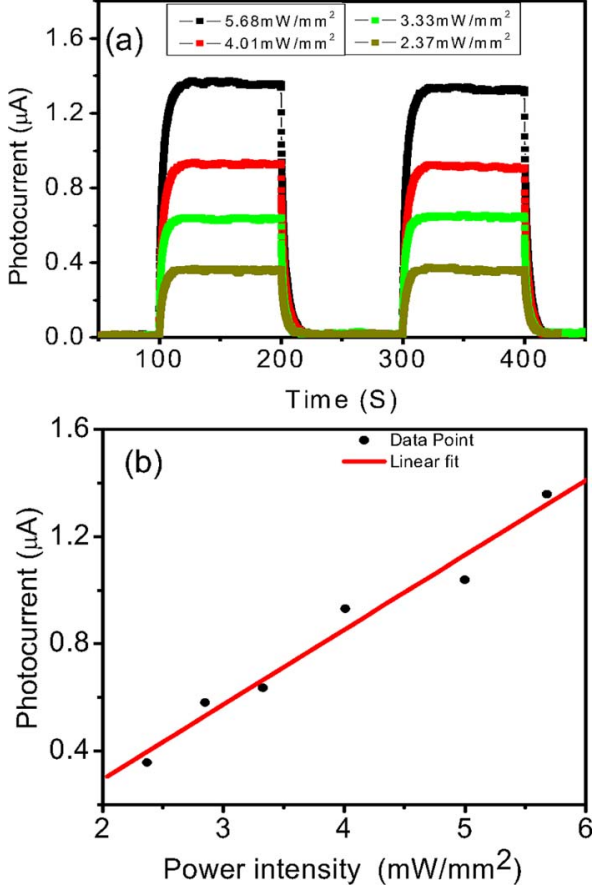

FIG. 3. (Color online) (a) Photocurrent vs time for a few different laser intensities. (b) Dependence of photocurrent of the MWNT film on the laser intensity.

CNTs and generate more excitons. So a greater number of electrons have the probability to overcome the Schottky barrier, generating a larger photovoltage. On the other hand, when the intensity of laser light is low, a smaller photovoltage is generated.

We now investigate the time response of the photocurrent. Figure 4(a) shows a plot of the rising part (first $40 \mathrm{~s}$ ) of the normalized photocurrent $\left(I / I_{\max }\right)$ versus time for all of our MWNT films with electrode separations of 2, 3, 5, 10, $20,25,40$, and $50 \mathrm{~mm}$ when illuminated at the left electrode. The top curve is for $d=2 \mathrm{~mm}$ and the bottom is for $d$ $=50 \mathrm{~mm}$. Two features can be noticed from these data: (i) the time taken to reach maximum photocurrent (response time) is rather slow and (ii) the response time increases with increasing separation. In order to determine whether the slow time response had indeed come from the NIR source illumination and not a delay due to a $R$ - $C$-like circuit existing in the entire setup, we measured the response of one of our films with a step function voltage (not shown here). We found that unlike NIR source, the current increased almost instantaneously to a bias voltage switch. Therefore, the slow time response indeed comes from the NIR illumination and not a delay caused by a $R$ - $C$-like circuit existing in the entire setup. The dynamic response to the NIR source can be well described by $I=I_{0}\left\{1-\exp \left[-\left(t-t_{0}\right) / \tau\right]\right\}$, where $\tau$ is the time constant, $t_{0}$ is the time when NIR is switched on, and $I_{0}$ is the steady state photocurrent. Figure 4(b) shows a fit of this equation for one of the sample with electrode separation of $10 \mathrm{~mm}$. Open circles are the experimental points and the solid line is a fit to the above equation. From this fit we obtain $\tau=2.65$ s. Similar fits were done for all the samples and the measured time constants were $0.35,0.62,1.50,2.65$, $3.39,4.13$, and $5.27 \mathrm{~s}$ for $2,3,5,10,20,25,40$, and $50 \mathrm{~mm}$
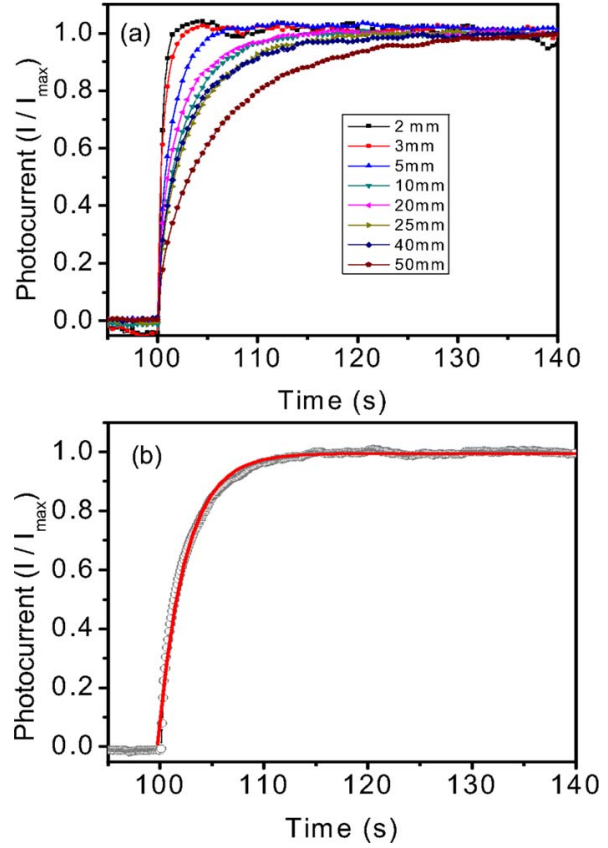

FIG. 4. (Color online) Time response of the photocurrent. (a) Raising part of normalized photocurrent as a function of time for all film with electrode separations of $2-50 \mathrm{~mm}$. The laser light was positioned at left MWNTs/ electrode interface. (b) Sample with electrode separation $10 \mathrm{~mm}$. The open circles are data and the solid line is the exponential fit with a time constant $\tau=2.65 \mathrm{~s}$.

electrode separations, respectively. From here, we conclude that the time constant increases with increasing electrode separations. Similar increases in time constants were also obtained for the decaying part when the laser was switched off and for the right electrode-CNT film interface.

There is a lot of debate about the origin of slow time response of photocurrent in CNT films. Previous studies in SWNT films have shown that bolometric effect, ${ }^{3}$ molecular photodesorption, ${ }^{7}$ and charge carrier diffusion ${ }^{12}$ can explain the photoresponse and slow time response in photocurrent. In bolometric mechanism, the temperature of the CNT film increases upon NIR source illumination causing a decrease in resistance, hence generating only a positive photoresponse. While in photodesorption effect, there is also a positive photoresponse and an increasing drift current over time. However, in our experiment, we found both positive and negative photoresponse at two different interfaces $\mathrm{L}$ and $\mathrm{R}$, respectively. In addition, our data are highly reproducible and we did not find any drift current over time for several cycles (Fig. 2). So, we rule out both bolometric and photodesorption mechanisms in our films. We now examine whether carrier diffusion model can explain our data. According to the diffusion model, considering a parabolic impurity density distribution, the time constant can be described as ${ }^{12,19}$

$$
\tau=\frac{d^{2}}{2 D_{p}(r+1)}\left[1-\frac{I_{(1 / 2)(r+3)}\left(d / L_{p}\right)}{I_{(1 / 2)(r-1)}\left(d / L_{p}\right)}\right],
$$

where $d$ is the electrode separation, $L_{p}$ is the diffusion length, $D_{p}$ is the diffusion coefficient of hole, $I_{n}$ is the modified Bessel of the first kind of order $n$, and $r \sim d^{\alpha}$ is a real constant for a parabolic impurity density distribution function. 


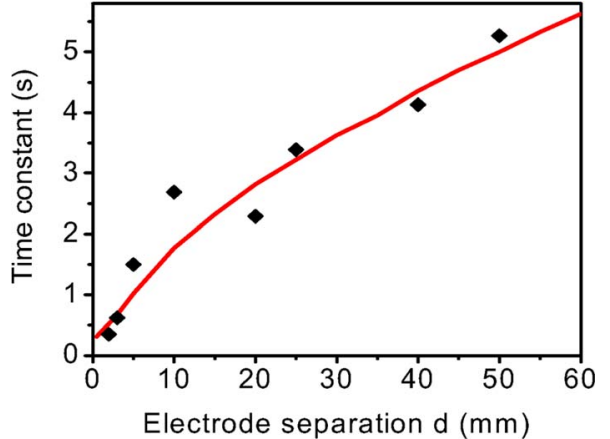

FIG. 5. (Color online) Variation in time constant with electrode separation. The black squares are the measured time constant and the solid line is a fit to the diffusion model.

According to this model, the time constant should increase with increasing electrode separations. In order to see whether the diffusion model can describe the slow time response in our MWNT networks, we plotted the time constant as a function of electrodes separation in Fig. 5 and fitted the data with the above equation. The black squares are the measured time constants, while the solid curve is a fit to the diffusion equation for charge carrier using $L_{p}=1 \mathrm{~mm}, D_{p}=0.01 \mathrm{~cm}^{2} / \mathrm{V} \mathrm{s}$ as fixed and keeping $\alpha$ as a free parameter. We obtained $\alpha$ $=1.4$ from the fit. We chose similar values for the diffusion length and diffusion coefficient as in SWNT films ${ }^{12}$ because, like SWNT film, the charge transport in MWNT film is also dominated by internanotube junctions and not by the ballistic nature of individual nanotubes. It can be seen from Fig. 5 that the experimental data can be fitted reasonably well with the diffusion model. Therefore, we conclude that the slow time response in our film is due to the diffusion of free charge carriers that was created at the metal-MWNT film interface. In other words, the slow response is due to the diffusion mediated charge transport through many interconnected individual MWNTs.

\section{CONCLUSIONS}

In conclusion, we presented NIR photoresponse study of MWNT films with various electrode separations. We found that the photoresponse is position-dependent with highest photoresponse occurring at MWNT/metal interface and is consistent with the model of Schottky barrier modulation for photocurrent generation. The time constant for dynamic photoresponse increases with increasing electrode separations and can be explained by the diffusion of charge carriers through percolating MWNT interconnects. Our results presented here will have important implications on the use of MWNT thin films for photodetectors and photovoltaic devices.

\section{ACKNOWLEDGMENTS}

This work is supported in part by U.S. National Science Foundation under Grant No. ECCS 0801924.

${ }^{1}$ P. Avouris, Z. H. Chen, and V. Perebeinos, Nat. Nanotechnol. 2, 605 (2007).

${ }^{2}$ R. H. Baughman, A. A. Zakhidov, and W. A. de Heer, Science 297, 787 (2002).

${ }^{3}$ M. E. Itkis, F. Borondics, A. Yu, and R. C. Haddon, Science 312, 413 (2006).

${ }^{4}$ J. L. Sun, J. Xu, J.-L. Zhu, and B. Li, Appl. Phys. A: Mater. Sci. Process. 91, 229 (2008)

${ }^{5}$ M. W. Rowell, M. A. Topinka, and M. D. McGehee, Appl. Phys. Lett. 88, 233506 (2006)

${ }^{6}$ A. D. Pasquier, H. E. Unalan, and A. Kanwal, Appl. Phys. Lett. 87, 203511 (2005)

${ }^{7}$ I. A. Levitsky and W. B. Euler, Appl. Phys. Lett. 83, 1857 (2003).

${ }^{8}$ B. Pradhan, K. Setyowati, H. Liu, D. H. Waldeck, and J. Chen, Nano Lett. 8, 1142 (2008).

${ }^{9}$ S. Lu and B. Panchapakesan, Nanotechnology 17, 1843 (2006).

${ }^{10}$ D.-H. Lien, W.-K. Hsu, H.-W. Zan, H.-H. Tai, and C.-H. Tsai, Adv. Mater. 18, 98 (2006).

${ }^{11}$ Y. Liu, S. Lu, and B. Panchapakesan, Nanotechnology 20, 035203 (2009).

${ }^{12}$ C. A. Merchant and N. Markovića, Appl. Phys. Lett. 92, 243510 (2008).

${ }^{13}$ C. A. Merchant and N. Markovića, Nanotechnology 20, 175202 (2009).

${ }^{14}$ J.-L. Sun, J. Wei, J.-L. Zhu, D. Xu, X. Liu, H. Sun, D.-H. Wu, and N.-L. Wu, Appl. Phys. Lett. 88, 131107 (2006).

${ }^{15}$ P. Stokes, L. Liu, J. Zou, L. Zhai, Q. Huo, and S. I. Khondaker, Appl. Phys. Lett. 94, 042110 (2009)

${ }^{16}$ P. Castrucci, F. Tombolini, M. Scarselli, E. Speiser, S. Del Gobbo, W. Richter, and M. De Crescenzi, Appl. Phys. Lett. 89, 253107 (2006).

${ }^{17}$ M. Ahlskog, O. Herranen, A. Johansson, J. Lepaniemi, and D. Mtsuko, Phys. Rev. B 79, 155408 (2009).

${ }^{18}$ M. J. O'Connell, Carbon Nanotubes: Properties and Application (CRC Press, London, 2006), p. 22.

${ }^{19}$ A. E. Mostafa and W. A. Wassef, IEEE Trans. Electron Devices 17, 53 (1970). 